\title{
Safety of Polyethylene Glycol Solution plus Ascorbic Acid for Bowel Preparation for Colonoscopy in Patients with Chronic Kidney Disease
}

\author{
Naoki Ohmiya $(\mathbb{D}$, Yoshihito Nakagawa, Noriyuki Horiguchi, Takafumi Omori, \\ Toshiaki Kamano, Kohei Funasaka, Mitsuo Nagasaka, and Tomoyuki Shibata
}

Department of Gastroenterology, Fujita Health University School of Medicine, Toyoake, Aichi, Japan

Correspondence should be addressed to Naoki Ohmiya; nohmiya@med.nagoya-u.ac.jp

Received 3 November 2020; Revised 8 February 2021; Accepted 27 February 2021; Published 16 March 2021

Academic Editor: Naohisa Yoshida

Copyright (c) 2021 Naoki Ohmiya et al. This is an open access article distributed under the Creative Commons Attribution License, which permits unrestricted use, distribution, and reproduction in any medium, provided the original work is properly cited.

\begin{abstract}
Introduction. Polyethylene glycol-electrolyte lavage solution plus ascorbic acid (PEG-ELS-Asc) has been recommended for colonoscopy, but little is known about the safety of PEG-ELS-Asc in patients with chronic kidney disease (CKD). The aim of this study was to determine its safety and efficacy in CKD patients. Methods. Blood and urine samples prospectively collected before and after same-day bowel preparation for colonoscopy with the conventional volume of PEG-ELS-Asc, vital signs before and after colonoscopy, and adverse events within 30 days postcolonoscopy were analyzed in consenting patients with CKD. The cleansing level was evaluated with the Boston bowel preparation score (BBPS) from colonoscopic findings. Results. Of 57 patients enrolled, 1 was excluded for refusal. Serum bicarbonate significantly dropped, and blood hemoglobin, serum total protein, albumin, aspartate aminotransferase, alanine aminotransferase, lactate dehydrogenase, total bilirubin, and uric acid significantly rose after bowel preparation, although these changes were not clinically important. Only in nondialysis patients did the platelet count and potassium significantly rise, although these changes were not clinically important either. Renal function, such as the urea, creatinine, and estimated glomerular filtration rate, was not significantly altered. An adequate bowel cleansing score, BBPS $\geq 6$, was achieved in $94 \%$ of patients. The blood pressure and heart rate were not significantly different between before and after colonoscopy in either nondialysis $(n=32)$ or dialysis $(n=19)$ patients. There were no adverse events associated with bowel preparation and colonoscopy within 30 days postcolonoscopy. Conclusions. The conventional volume of same-day bowel preparation with PEG-ELS-Asc may be safe and effective in CKD patients.
\end{abstract}

\section{Introduction}

Polyethylene glycol-electrolyte lavage solution (PEG-ELS) has been a recommended cleansing agent for colonoscopy. As it is isoosmotic, PEG-ELS is considered a preferred agent in patients who are less likely to tolerate fluid intake, including patients with renal dysfunction, congestive heart failure, and cirrhosis. Use of a split-dose regimen of 4 L PEG-ELS is recommended for scheduled colonoscopy, and a same-day regimen is an acceptable alternative to split dosing, especially for patients undergoing colonoscopy in the afternoon [1]. Drinking such a large volume of PEG-ELS, however, induces poor patient compliance [2]. Another 2L PEG-ELS agent consists of supplemental ascorbic acid and sodium sulfate but no bicarbonate. The addition of ascorbic acid and sodium $\mathrm{L}$-ascorbate reduces the intake volume to only $2 \mathrm{~L}$ or less [3]. The cathartic effect of ascorbic acid is considered to be ascribed to its absorption mechanism, which becomes saturated at high doses $[4,5]$. Excess unabsorbed ascorbic acid remains in the bowel lumen where it exerts an osmotic effect, acting synergistically with PEG. PEG-ELS plus ascorbic acid (PEG-ELS-Asc) achieved successful gut cleansing similar to 4 L PEG-ELS and better acceptability [3], but it is hyperosmotic, which can induce dehydration and electrolyte disturbance. To our knowledge, little is known about the safety of PEG-ELS-Asc in patients with chronic kidney disease (CKD) $[6,7]$. In this study, we prospectively determined the safety of bowel cleansing with PEG-ELS-Asc before 
TABLE 1: Measurement parameters before and after bowel preparation for colonoscopy.

\begin{tabular}{|c|c|}
\hline Vital signs & Blood pressure, heart rate \\
\hline Urine examination & $\mathrm{Na}, \mathrm{K}, \mathrm{Cr}, \mathrm{RBC}, \mathrm{WBC}$, osmotic pressure \\
\hline Blood examination & CBC, $\mathrm{Na}, \mathrm{K}, \mathrm{Cl}, \mathrm{Ca}, \mathrm{P}, \mathrm{Mg}, \mathrm{BUN}, \mathrm{Cr}$, eGFR, TP, Alb, AST, ALT, LD, TBil, UA, $\mathrm{HCO}_{3}{ }^{-}$, osmolarity \\
\hline
\end{tabular}

Na: sodium; K: potassium; Cr: creatinine; RBC: red blood cell: WBC: white blood cell; CBC: complete blood count; Ca: calcium; P: phosphate; Mg: magnesium; BUN: blood urea nitrogen; eGFR: estimated glomerular filtration rate; TP: total protein; Alb: albumin; AST: aspartate aminotransferase; ALT: alanine aminotransferase; LD: lactate dehydrogenase; TBil: total bilirubin; DBil: direct bilirubin; CRP: C-reactive protein; UA: uric acid; $\mathrm{HCO}_{3}$ : bicarbonate.

colonoscopy in 51 patients with CKD by comparing the blood and urine samples before and after the bowel preparation and by a 30-day postcolonoscopy chart review.

\section{Methods}

2.1. Study Design and Patients. This was a prospective singlecenter observational study of adverse events of the same-day conventional volume of PEG-ELS-Asc (MoviPrep ${ }^{\circledR}$, EA Pharma Co., Ltd., Tokyo, Japan) by comparing the blood and urine examinations before and after bowel cleansing for conventional colonoscopy in patients with CKD. Outpatients and inpatients aged 20 years or older who were treated with $\mathrm{CKD}$ and required to undergo complete colonoscopy were recruited to the study between February 2015 and October 2016. The primary endpoint included clinically significant changes in urine and blood examinations including renal function. Secondary endpoints included changes in vital signs, cleansing levels at colonoscopy, and adverse events within 30 days postcolonoscopy. A sample size of 50 patients with CKD was planned to consider the risk of conventional 2 L PEG-ELS-Asc according to the previous report that same-day low-volume $1 \mathrm{~L}$ PEG-ELS-Asc showed significant elevation of serum creatinine concentration in 25 hemodialysis patients and $68 \mathrm{CKD}$ patients without hemodialysis [6]. All patients provided written informed consent, and this study was reviewed and approved by the Institutional Review Board of Fujita Health University School of Medicine. Consenting patients were included unless they had bowel obstruction or allergy to drugs used. Women were excluded from the study if they were pregnant or at risk of becoming pregnant. Patients with phenylketonuria and glucose-6-phosphate dehydrogenase deficiency were also excluded due to the presence of aspartame and ascorbate, respectively. This study was registered with the University Hospital Medical Information Network (UMIN000013041). All methods were carried out in accordance with relevant guidelines and regulations.

2.2. Definition of Chronic Kidney Disease. CKD was defined as abnormalities of kidney structure or function, present for $>3$ months, with implications for health, and CKD was classified based on the cause and glomerular filtration rate (GFR) category [8]. GFR was estimated using the revised equations from serum creatinine for Japanese patients [9]. The estimated GFR (eGFR) was classified into 6 grades: G1: $\geq 90$; G2: 60-89; G3a: 45-59; G3b: 30-44; G4: 15-29; and G5: $<15 \mathrm{~mL} / \mathrm{min} / 1.73 \mathrm{~m}^{2}$. G5D corresponds to G5 undergoing dialysis.
2.3. Bowel Preparation. We used the same-day regimen with PEG-ELS-Asc for colonoscopy in the afternoon. Bowel preparation and colonoscopy were performed on nondialysis days in dialysis patients. On the day before examination, all patients had three meals consisting of a low-fiber diet and fasted from $9 \mathrm{pm}$, and patients with constipation took sennoside. On the day of examination, all patients drank $1,000 \mathrm{~mL}$ of PEG-ELS-Asc over 1-1.5 h and then $500 \mathrm{~mL}$ of water or clear liquid-like tea. Experienced medical staff assessed the quality of the bowel preparation by checking the clarity of patients' evacuation. When the preparation quality was poor, patients drank another $500 \mathrm{~mL}$ PEG-ELS-Asc for $30 \mathrm{~min}$, then $250 \mathrm{~mL}$ of water or clear liquid. When the preparation quality was still poor, patients drank another $500 \mathrm{~mL}$ PEG-ELS-Asc for $30 \mathrm{~min}$ and then $250 \mathrm{~mL}$ of water or clear liquid again.

2.4. Measurement Parameters. Vital signs, including the blood pressure and heart rate, were recorded before and after colonoscopy on the examination table in the endoscopy center. Blood and urine were sampled before preparation and after colonoscopy within two hours. Urine was sampled from all patients except on dialysis without urination. The measured parameters of blood and urine samples are shown in Table 1.

2.5. Colonoscopy Procedure. A conventional colonoscope (CFHQ290I, PCF-Q260AZI, and PCF-H290I, Olympus Corporation, Tokyo, Japan; EC-L590ZW, Fujifilm Co., Ltd., Tokyo, Japan) was used for all colonoscopic examinations. When colorectal polyps or tumors were observed, endoscopic resection including polypectomy and endoscopic mucosal resection (EMR) was performed if possible without hospitalization. Most procedures were performed with air insufflation but $\mathrm{CO}_{2}$ insufflation in some cases. Conscious sedation was performed, mostly with midazolam $(2-10 \mathrm{mg})$, according to the patient's request or pain status in previous procedures. Antispasmodics such as scopolamine butylbromide or glucagon were administered depending upon the colonoscopist's decision. Monitoring with an automatic blood pressure monitor was performed in all cases during sedation.

2.6. Cleansing Levels at Colonoscopy. The cleansing level was evaluated in the right colon, transverse colon, and left colon with the Boston bowel preparation score (BBPS) from colonoscopic findings [10]. The total score of the BBPS was categorized into two groups: $6-9$ and $0-5$. BBPS $\geq 6$ was defined as good preparation, and BBPS 0-5 was defined as poor preparation.

2.7. Statistical Analysis. The numbers in the text are expressed as the median and range or mean and standard 
TABLE 2: Clinical characteristics of patients with chronic kidney disease.

\begin{tabular}{|c|c|c|}
\hline Characteristics & Total & $\%$ \\
\hline No. & 56 & \\
\hline \multicolumn{3}{|l|}{ Age (years) } \\
\hline Mean & 65 & \\
\hline Standard deviation & 12 & \\
\hline \multicolumn{3}{|l|}{ Sex } \\
\hline Male & 38 & $68 \%$ \\
\hline Female & 18 & $32 \%$ \\
\hline \multicolumn{3}{|l|}{ Healthcare services } \\
\hline Outpatient & 32 & $57 \%$ \\
\hline Inpatient & 24 & $43 \%$ \\
\hline \multicolumn{3}{|l|}{ Height (cm) } \\
\hline Mean & 159.6 & \\
\hline Standard deviation & 9.0 & \\
\hline \multicolumn{3}{|l|}{ Body weight (kg) } \\
\hline Mean & 59.5 & \\
\hline Standard deviation & 13.5 & \\
\hline \multicolumn{3}{|l|}{ Body mass index } \\
\hline Mean & 23.2 & \\
\hline Standard deviation & 4 & \\
\hline \multicolumn{3}{|l|}{ Cause of chronic kidney disease } \\
\hline Diabetic nephropathy & 20 & $36 \%$ \\
\hline Hypertensive nephrosclerosis & 16 & $29 \%$ \\
\hline Membranous nephropathy & 4 & $7 \%$ \\
\hline Polycystic kidney & 2 & $4 \%$ \\
\hline Tubulointerstitial nephritis & 2 & $4 \%$ \\
\hline Minimal change nephrotic syndrome & 1 & $2 \%$ \\
\hline IgA nephropathy & 1 & $2 \%$ \\
\hline Sarcoidosis-related tubulointerstitial nephritis & 1 & $2 \%$ \\
\hline Lupus nephritis & 1 & $2 \%$ \\
\hline Fanconi syndrome & 1 & $2 \%$ \\
\hline Ischemic nephropathy & 1 & $2 \%$ \\
\hline Reflux nephropathy & 1 & $2 \%$ \\
\hline $\begin{array}{l}\text { Postnephrectomy and chemotherapy for renal } \\
\text { pelvis cancer }\end{array}$ & 1 & $2 \%$ \\
\hline Kidney transplant recipient & 1 & $2 \%$ \\
\hline Miscellaneous & 3 & $5 \%$ \\
\hline \multicolumn{3}{|l|}{ Classification of chronic kidney disease } \\
\hline G2 (eGFR: 60-89) & 1 & $2 \%$ \\
\hline G3a (eGFR: 45-59) & 4 & $7 \%$ \\
\hline G3b (eGFR: 30-44) & 10 & $18 \%$ \\
\hline G4 (eGFR: 15-29) & 6 & $11 \%$ \\
\hline G5 (eGFR: <15) & 14 & $25 \%$ \\
\hline G5D (eGFR: <15, dialysis) & 21 & $38 \%$ \\
\hline \multicolumn{3}{|l|}{ Indications } \\
\hline FIT positive & 24 & $43 \%$ \\
\hline Melena or hematochezia & 6 & $11 \%$ \\
\hline Follow-up & 6 & $11 \%$ \\
\hline Anemia & 3 & $5 \%$ \\
\hline
\end{tabular}

TABLe 2: Continued.

\begin{tabular}{|c|c|c|}
\hline Characteristics & Total & $\%$ \\
\hline Diarrhea or constipation & 4 & $7 \%$ \\
\hline Abdominal pain & 1 & $2 \%$ \\
\hline Other screening* & 12 & $21 \%$ \\
\hline \multicolumn{3}{|l|}{ Medication } \\
\hline Diuretics & 32 & $57 \%$ \\
\hline Polystyrene cation-exchange resin & 3 & $5 \%$ \\
\hline \multicolumn{3}{|l|}{ Previous laparotomy } \\
\hline Presence & 11 & $22 \%$ \\
\hline Absence & 45 & $88 \%$ \\
\hline \multicolumn{3}{|c|}{$\begin{array}{l}\text { deviation, depending upon the fit of a normal distribution. } \\
\text { The numbers were tested for normality using the D'Agostino } \\
\text { test, the Shapiro-Wilk test, and the Kolmogorov-Smirnov } \\
\text { test. Comparisons were analyzed between the variables } \\
\text { before and after bowel preparation or colonoscopy using } \\
\text { the Wilcoxon signed rank test or the paired } t \text {-test. When } \\
\text { the cohort population was less than } 30 \text {, the Wilcoxon signed } \\
\text { rank test was used. Differences were considered significant } \\
\text { with } P \text { values less than } 0.05 \text {. }\end{array}$} \\
\hline
\end{tabular}

\section{Results}

3.1. Patients with Chronic Kidney Disease. Of 57 patients with CKD enrolled between February 2015 and October 2016, 1 was excluded because he refused bowel preparation and colonoscopy. Table 2 shows the clinical characteristics of the remaining 56 patients. Five patients did not undergo blood examination after colonoscopy. Regarding drugs associated with hypokalemia, diuretics and polystyrene cation-exchange resin were used in 32 (57\%) and 3 (5\%) patients, respectively. Of 32 patients on these drugs, 3 took diuretics and the remaining 29 discontinued the drugs on the day of colonoscopy.

3.2. Colonoscopy. Of 56 patients, 27 underwent colonoscopy without biopsy or endoscopic treatment, 5 underwent colonoscopy with biopsy, and 24 underwent colonoscopy with endoscopic polyp resection. Table 3 shows the volume of bowel preparation and Boston bowel preparation score.

\subsection{Measurement Parameters}

3.3.1. Blood Samples. Tables 4 and 5 show the blood parameters before and after bowel preparation in all $(n=51)$, nondialysis $(n=32)$, and dialysis patients $(n=19)$. In both the nondialysis and dialysis patients, bicarbonate significantly dropped, and hemoglobin, total protein, albumin, aspartate aminotransferase (AST), alanine aminotransferase (ALT), lactate dehydrogenase (LD), total bilirubin, and uric acid significantly rose, although these changes were clinically insignificant. Only in nondialysis patients did the platelet count and potassium significantly rise, although these changes were also clinically insignificant. Renal function, such as urea, creatinine, and eGFR, was not significantly changed either in nondialysis patients or in dialysis patients. 
TABLE 3: Bowel preparation and colonoscopy.

\begin{tabular}{|c|c|}
\hline Variables & $\begin{array}{c}\text { Total } \\
(\%)\end{array}$ \\
\hline \multicolumn{2}{|c|}{$\begin{array}{l}\text { Polyethylene glycol-electrolyte lavage solution plus } \\
\text { ascorbic acid (mL) }\end{array}$} \\
\hline Mean & 1,496 \\
\hline Standard deviation & 381 \\
\hline \multicolumn{2}{|l|}{ Water or clear liquid (mL) } \\
\hline Median & 750 \\
\hline Range & $0-2,000$ \\
\hline \multicolumn{2}{|c|}{$\begin{array}{l}\text { Time from the beginning of bowel preparation to clear } \\
\text { evacuation ( } \mathrm{min})\end{array}$} \\
\hline Median & 182.5 \\
\hline Range & $50-542$ \\
\hline \multicolumn{2}{|l|}{ Boston bowel preparation score } \\
\hline \multicolumn{2}{|l|}{ Total score } \\
\hline Median & 8 \\
\hline Range & $2-9$ \\
\hline Good preparation (6-9) & $\begin{array}{c}52 \\
(93 \%)\end{array}$ \\
\hline Poor preparation $(0-5)$ & $4(7 \%)$ \\
\hline \multicolumn{2}{|l|}{ Right colon } \\
\hline Median & 2 \\
\hline Range & $0-3$ \\
\hline \multicolumn{2}{|l|}{ Transverse colon } \\
\hline Median & 3 \\
\hline Range & $1-3$ \\
\hline \multicolumn{2}{|l|}{ Left colon } \\
\hline Median & 3 \\
\hline Range & $1-3$ \\
\hline
\end{tabular}

In 35 patients without the use of sedatives or antispasmodics during colonoscopy, AST, ALT, LD, and total bilirubin were significantly changed: 15 to $19 \mathrm{IU} / \mathrm{L}, 11$ to $14 \mathrm{IU} / \mathrm{L}$, 200 to $215 \mathrm{IU} / \mathrm{L}$, and 0.5 to $0.6 \mathrm{mg} / \mathrm{dL}$ (median, $P<0.001$, $<$ $0.001,<0.001$, and 0.002 ), respectively. In 16 patients with the use of sedatives or antispasmodics during colonoscopy, AST and ALT were significantly changed: 17.5 to $19.5 \mathrm{IU} / \mathrm{L}$ and 10 to $12 \mathrm{IU} / \mathrm{L}$ (median, $P=0.004$ and 0.022 ), respectively, while changes in LD and total bilirubin were not significant (median, 208 to $224 \mathrm{IU} / \mathrm{L}$ and 0.5 to $0.5 \mathrm{mg} / \mathrm{dL}$, $P=0.061$ and 0.244 , respectively). In 29 patients who underwent colonoscopy without any endoscopic intervention except biopsy, AST, ALT, LD, and total bilirubin were significantly changed: 17 to $20 \mathrm{IU} / \mathrm{L}, 10$ to $13 \mathrm{IU} / \mathrm{L}, 207$ to $226 \mathrm{IU} / \mathrm{L}$, and 0.5 to $0.6 \mathrm{mg} / \mathrm{dL}$ (median, $P<0.001,<0.001,<0.001$, and 0.007 ), respectively. In 22 patients who underwent colonoscopy with endoscopic intervention, AST and ALT were significantly changed: 15 to $18.5 \mathrm{IU} / \mathrm{L}$ and 11 to $13.5 \mathrm{IU} / \mathrm{L}$ (median, $P=0.002$ and 0.010 ), respectively, while changes in LD and total bilirubin were not significant (median, 198 to $210 \mathrm{IU} / \mathrm{L}$ and 0.5 to $0.5 \mathrm{mg} / \mathrm{dL}, P=0.251$ and 0.064 , respectively).

In a male nondialysis patient with nephrotic syndrome due to membranous nephropathy, transient liver injury developed: AST: 27 to $226 \mathrm{IU} / \mathrm{L}$; ALT: 29 to 153; LD: 200 to 394; and total bilirubin: 0.3 to 0.4 . He had been treated with $30 \mathrm{mg}$ of prednisolone for 7 days before colonoscopy with endoscopic resection of three polyps in addition to prophylactic administration of trimethoprim-sulfamethoxazole starting in the morning of the examination day. During colonoscopy, he took no medications including sedatives or antispasmodics. Even though trimethoprim-sulfamethoxazole was continued thereafter, liver injury was spontaneously recovered as follows: AST: 64 and 17; ALT: 101 and 37; and LD: 218 and 194 on postcolonoscopy days 1 and 4, respectively, which may have been ascribed to PEG-ELS-Asc.

Serum potassium levels significantly increased after bowel preparation only in nondialysis patients but not in dialysis patients. Serum potassium levels tended to increase in nondialysis patients on diuretics and not on diuretics (median, 4.25 to $4.50 \mathrm{mEq} / \mathrm{L}$ and 4.50 to $4.60 \mathrm{mEq} / \mathrm{L} ; P=$ 0.134 and 0.069 , respectively). Regarding the intake volume of PEG-ELS-Asc, median serum potassium levels before and after were 4.3 and $4.2 \mathrm{mEq} / \mathrm{L}$ in patients on $1.5 \mathrm{~L}$ or more, respectively $(P=0.566)$, and 4.95 to 4.85 in patients on less than $1.5 \mathrm{~L}$, respectively $(P=0.431)$.

3.3.2. Urine Samples. Table 4 shows the urine parameters before and after bowel preparation in all nondialysis and dialysis patients who could provide paired urine samples. Of 32 nondialysis and 19 dialysis patients, 30 (94\%) and 11 (58\%) provided urine samples, respectively. In nondialysis patients, urine potassium and osmolarity were significantly higher and urine chloride was significantly lower after bowel preparation with PEG-ELS-Asc than before. In dialysis patients, urine potassium was significantly higher after bowel preparation with PEG-ELS-Asc than before.

3.3.3. Vital Signs. Supplementary Table shows the blood pressure and heart rate before and after colonoscopy in all $(n=51$ ) , nondialysis $(n=32)$, and dialysis patients $(n=19)$. The systolic and diastolic blood pressure and heart rate did not significantly differ after colonoscopy from before colonoscopy in either nondialysis or dialysis patients.

3.3.4. Adverse Events within 30 Days Postcolonoscopy. Blood was sampled in 35 patients (69\%) within 30 days postcolonoscopy. Three patients developed aspiration pneumonia on the $12^{\text {th }}$ day (CKD stage G5A3), transient ischemic attack on the $20^{\text {th }}$ day (CKD stage G3aA3), and pulmonary edema on the $29^{\text {th }}$ day (CKD stage G5A3) postcolonoscopy, respectively. No nondialysis patients developed into a dialysis stage. There were no adverse events associated with bowel preparation and colonoscopy.

\section{Discussion}

The safety and usefulness of PEG-ELS have been described in many studies. The present study demonstrated that a conventional volume of PEG-ELS-Asc was safe for patients with $\mathrm{CKD}$ whether on dialysis or not. Yoshida et al. reported that a same-day low volume of $1 \mathrm{~L}$ PEG-ELS-Asc and $0.5 \mathrm{~L}$ water was safe in elderly patients aged $\geq 80$ years and patients with renal dysfunction in a single-center retrospective analysis, in 
TABLE 4: Comparison of blood and urine parameters before and after bowel preparation with polyethylene glycol solution plus ascorbic acid in all patients.

\begin{tabular}{|c|c|c|c|c|}
\hline \multirow{2}{*}{ Parameter } & \multirow{2}{*}{ Normal range } & \multicolumn{3}{|c|}{ All patients $(n=51)$} \\
\hline & & Before preparation & After preparation & $P$ \\
\hline \multicolumn{5}{|l|}{ Blood samples } \\
\hline White blood cell count $(/ \mu \mathrm{L})$ & $3,300-8,600$ & $5,800(2,900-16,100)$ & $6,800(2,900-25,600)$ & 0.035 \\
\hline Hemoglobin (g/dL) & $\begin{array}{l}13.7-16.8 \text { (male) } \\
11.6-14.8 \text { (female) }\end{array}$ & $11.0 \pm 1.5$ & $11.4 \pm 1.5$ & 0.001 \\
\hline Platelet count $\left(\times 10^{4} / \mathrm{mL}\right)$ & $15.8-34.8$ & $20.6(11.1-49.7)$ & $21.2(11.7-45.7)$ & 0.015 \\
\hline $\mathrm{Na}(\mathrm{mEq} / \mathrm{L})$ & $138-146$ & $140 \pm 3.7$ & $141 \pm 3.5$ & 0.060 \\
\hline $\mathrm{K}(\mathrm{mEq} / \mathrm{L})$ & $3.6-4.9$ & $4.5 \pm 0.7$ & $4.5 \pm 0.7$ & 0.088 \\
\hline $\mathrm{Cl}(\mathrm{mEq} / \mathrm{L})$ & $99-109$ & $103 \pm 4.8$ & $104 \pm 4.4$ & 0.076 \\
\hline $\mathrm{Ca}(\mathrm{mg} / \mathrm{dL})$ & $8.7-10.3$ & $9.2 \pm 0.8$ & $9.3 \pm 0.7$ & 0.217 \\
\hline $\mathrm{P}(\mathrm{mg} / \mathrm{dL})$ & $2.5-4.7$ & $4.1(2.7-7.5)$ & $4.4(2.2-9.2)$ & 0.068 \\
\hline $\mathrm{Mg}(\mathrm{mg} / \mathrm{dL})$ & $1.8-2.4$ & $2.0(1.1-3.1)$ & $2.0(1.1-3.4)$ & 0.341 \\
\hline BUN (mg/dL) & $8.0-22.0$ & $35.8(8.3-95.4)$ & $30.0(7.5-96.1)$ & 0.431 \\
\hline $\mathrm{Cr}(\mathrm{mg} / \mathrm{dL})$ & $0.6-1.1$ & $4.68 \pm 3.06$ & $4.89 \pm 3.23$ & 0.075 \\
\hline eGFR $\left(\mathrm{mL} / \mathrm{min} / 1.73 \mathrm{~m}^{2}\right)$ & $\geq 60.0$ & $10.1(3.8-76.4)$ & $9.5(3.5-84.2)$ & 0.376 \\
\hline $\mathrm{TP}(\mathrm{g} / \mathrm{dL})$ & $6.7-8.3$ & $6.8(3.9-8.1)$ & $7.3(4.7-8.3)$ & $<0.001$ \\
\hline $\mathrm{Alb}(\mathrm{g} / \mathrm{dL})$ & $4.0-5.0$ & $4.0(1.4-5.0)$ & $4.2(1.8-5.3)$ & $<0.001$ \\
\hline AST (IU/L) & $13-33$ & $16(3-50)$ & $19(4-226)$ & $<0.001$ \\
\hline $\operatorname{ALT}(\mathrm{IU} / \mathrm{L})$ & $6-30$ & $11(2-64)$ & $13(51-153)$ & $<0.001$ \\
\hline $\mathrm{LD}(\mathrm{IU} / \mathrm{L})$ & $119-229$ & $205(151-338)$ & $222(132-439)$ & $<0.001$ \\
\hline TBil (mg/dL) & $0.3-1.2$ & $0.5(0.2-1.2)$ & $0.5(0.2-1.3)$ & 0.001 \\
\hline $\mathrm{UA}(\mathrm{mg} / \mathrm{dL})$ & $3.6-7.0$ & $5.8 \pm 1.5$ & $6.2 \pm 1.5$ & 0.002 \\
\hline $\mathrm{HCO}_{3}^{-}(\mathrm{mmol} / \mathrm{L})$ & $20.0-30.0$ & $25.6 \pm 3.4$ & $23.3 \pm 3.3$ & $<0.001$ \\
\hline Osmolarity $\left(\mathrm{mOsm} / \mathrm{kgH}_{2} \mathrm{O}\right)$ & $275-290$ & $294(267-317)$ & $295(265-313)$ & 0.906 \\
\hline \multicolumn{5}{|l|}{ Urine samples } \\
\hline $\mathrm{Na}(\mathrm{mEq} / \mathrm{L})$ & ND & $78(16-147)$ & $69(6-190)$ & 0.882 \\
\hline $\mathrm{K}(\mathrm{mEq} / \mathrm{L})$ & ND & $22(4-71)$ & $36(4-91)$ & $<0.001$ \\
\hline $\mathrm{Cl}(\mathrm{mEq} / \mathrm{L})$ & ND & $71(10-151)$ & $52(10-138)$ & 0.030 \\
\hline Osmolarity $\left(\mathrm{mOsm} / \mathrm{kgH}_{2} \mathrm{O}\right)$ & $50-1300$ & $307(126-691)$ & $346(114-752)$ & 0.007 \\
\hline
\end{tabular}

Median (range); mean \pm standard deviation. ND: not determined; WBC: white blood cell; Na: sodium; K: potassium; Ca: calcium; P: phosphate; Mg: magnesium; BUN: blood urea nitrogen; Cr: creatinine; eGFR: estimated glomerular filtration rate; TP: total protein; Alb: albumin; AST: aspartate aminotransferase; ALT: alanine aminotransferase; LD: lactate dehydrogenase; TBil: total bilirubin; DBil: direct bilirubin; CRP: C-reactive protein; UA: uric acid; $\mathrm{HCO}_{3}^{-}$: bicarbonate.

which there were significant differences in blood hematocrit $(36.7 \pm 6.1 \%$ vs. $39.0 \pm 5.7 \%)$ and serum creatinine levels $(1.30 \pm 0.12 \mathrm{mg} / \mathrm{dL}$ vs. $1.37 \pm 0.14 \mathrm{mg} / \mathrm{dL})$ before and after bowel preparation in 68 renal dysfunction patients without hemodialysis (serum creatinine level, $1.1-2.9 \mathrm{mg} / \mathrm{mL}$ ), while there were no significant differences except for serum creatinine levels $(6.14 \pm 1.2 \mathrm{mg} / \mathrm{dL}$ vs. $6.80 \pm 1.4 \mathrm{mg} / \mathrm{dL})$ in 25 hemodialysis patients. Lee et al. reported that the laboratory findings were not significantly different before and after the administration of $2 \mathrm{~L}$ polyethylene glycol plus ascorbic acid or $4 \mathrm{~L}$ polyethylene glycol. In both groups, the estimated glomerular filtration rate was not influenced by the administration of the bowel cleansing agent [7]. The present study using a same-day regimen of the conventional volume of PEGELS-Asc (median volume 1.5 L of PEG-ELS-Asc and $750 \mathrm{~mL}$ of water or clear liquid) demonstrated that blood hemoglobin, serum total protein, albumin, AST, ALT, LD, and total bilirubin were significantly increased, and bicarbonate was significantly decreased after preparation in both the nondialysis and dialysis patients. The blood platelet count, serum potassium, and uric acid levels were increased only in nondialysis patients, while renal function represented by blood urea nitrogen, serum creatinine, and eGFR was not significantly worsened after bowel preparation in either nondialysis or dialysis patients. These elevated blood hemoglobin, serum total protein, and albumin levels were observed even in patients without comorbidities due to dehydration and hemoconcentration $[11,12]$ and showed subtle changes; therefore, they seemed clinically irrelevant. A significant decrease in serum sodium bicarbonate after the intake of PEG-ELS-Asc was also observed in patients without comorbidities [11]. PEG-ELS-Asc (MoviPrep) contains $11.8 \mathrm{~g}$ 


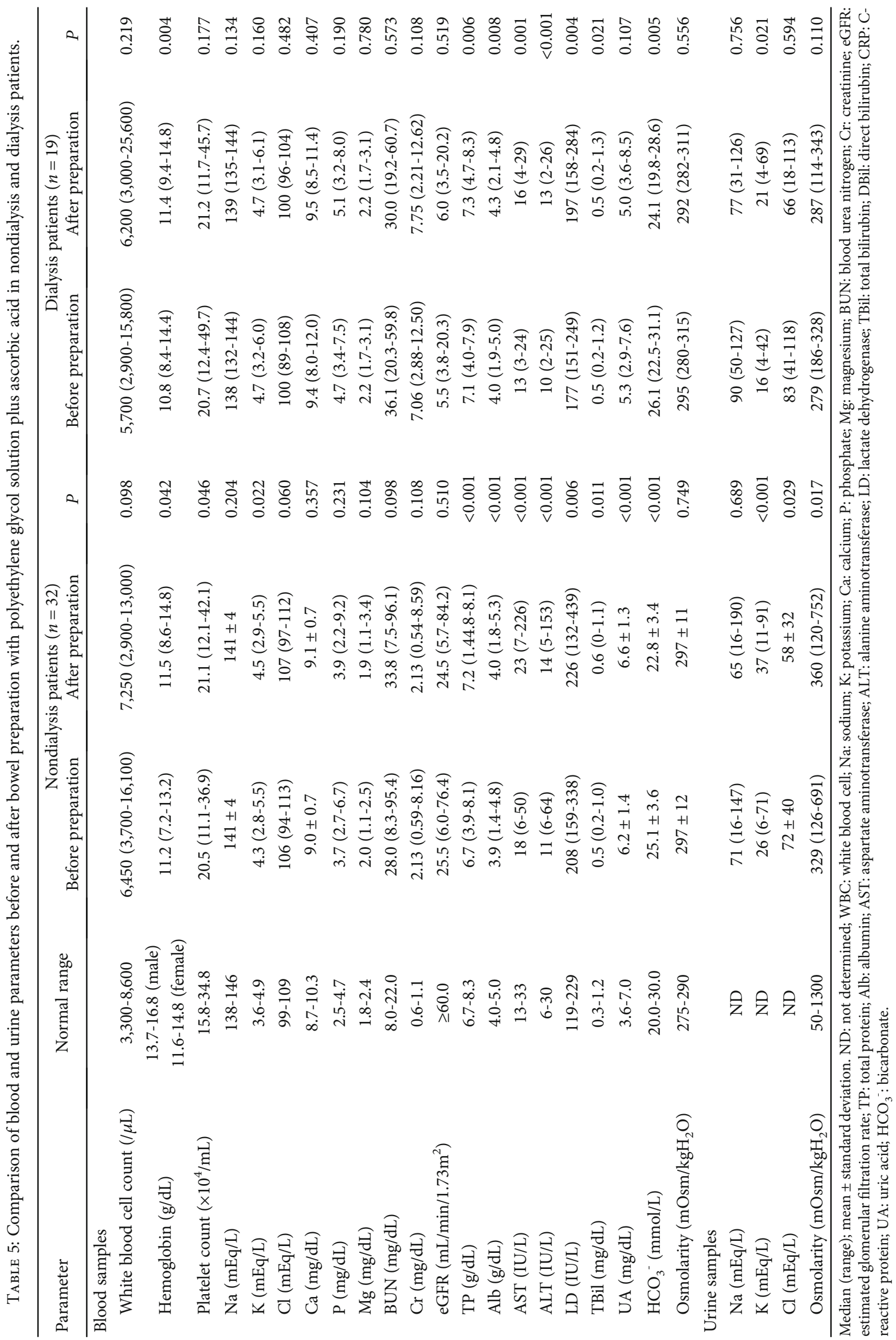

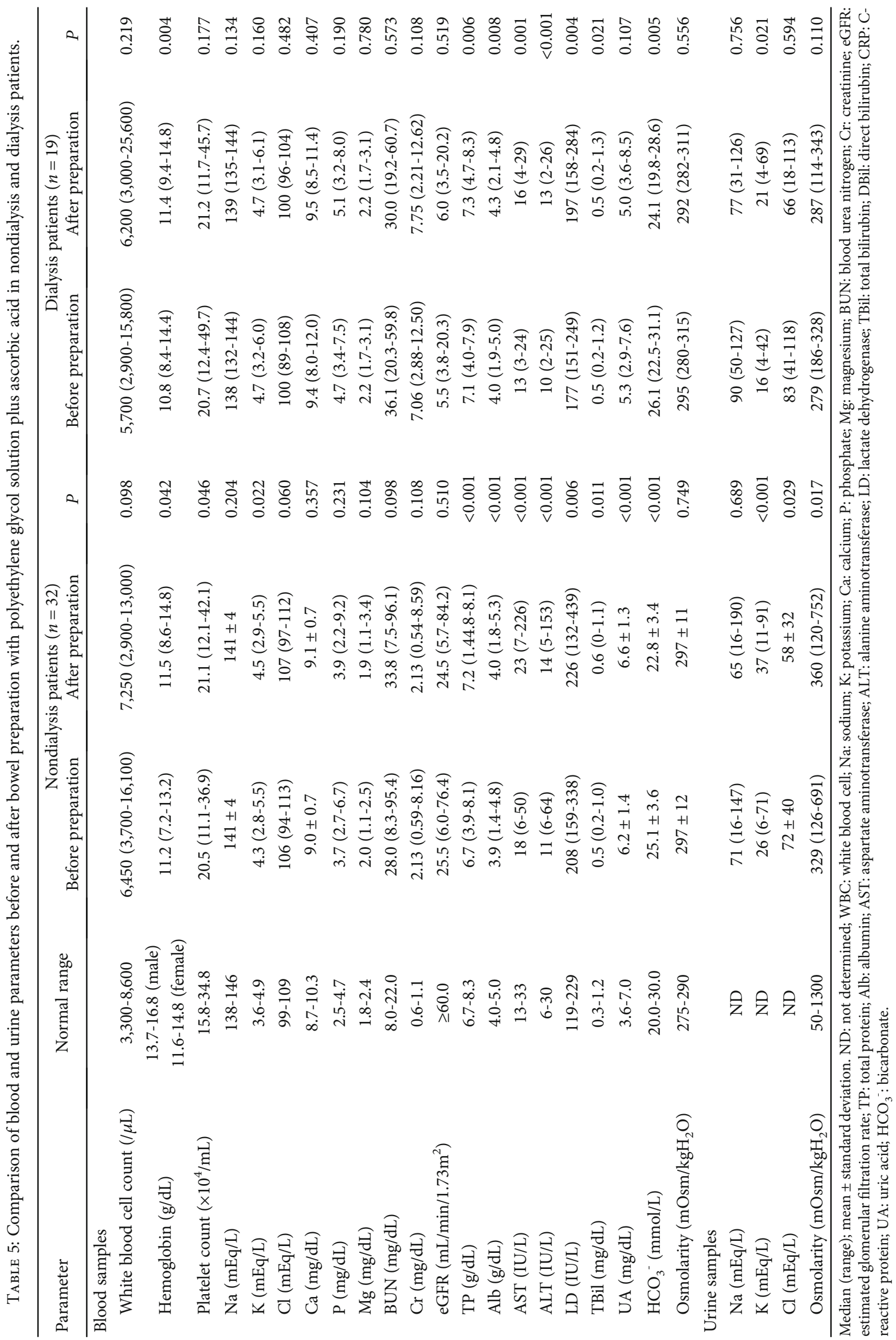

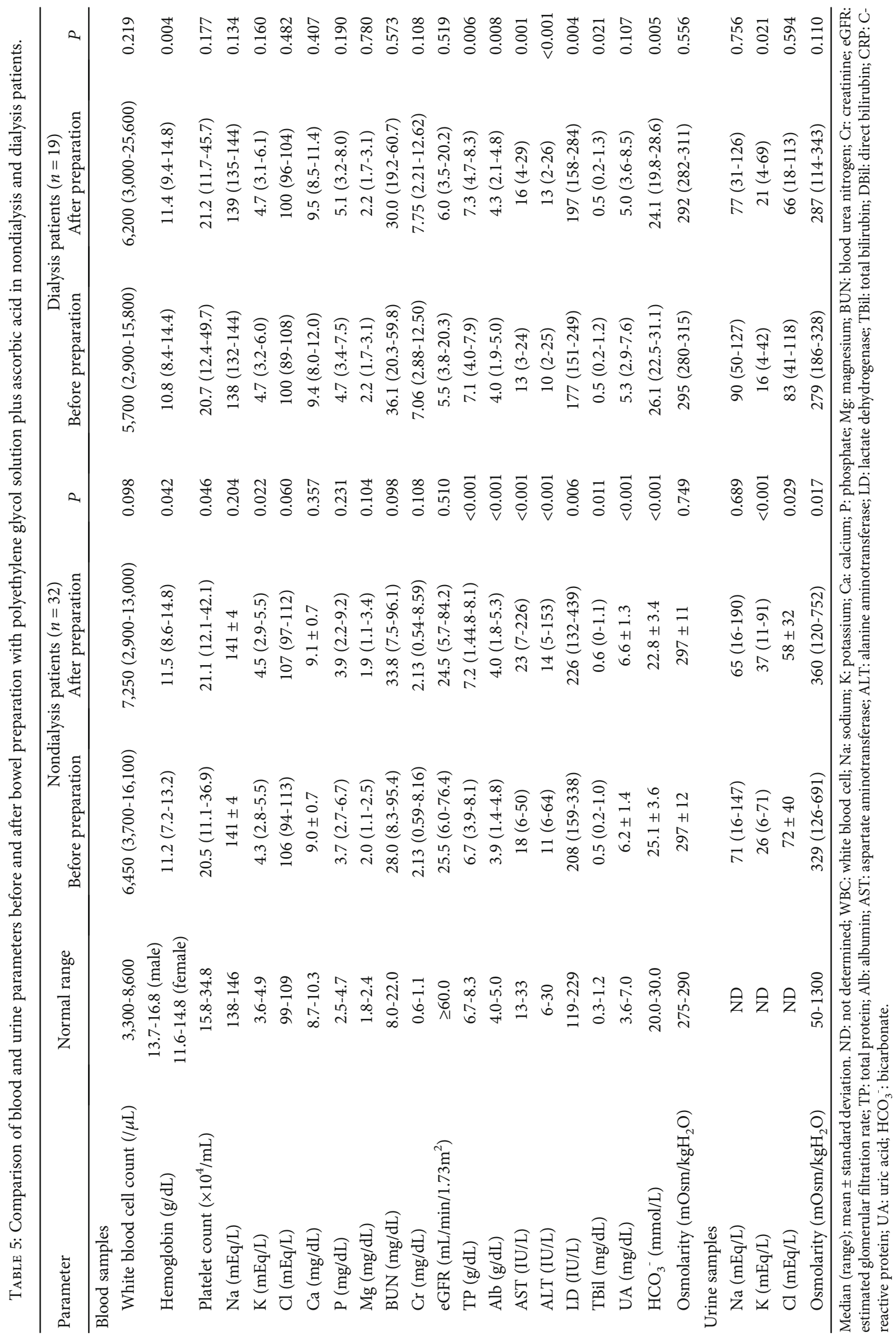

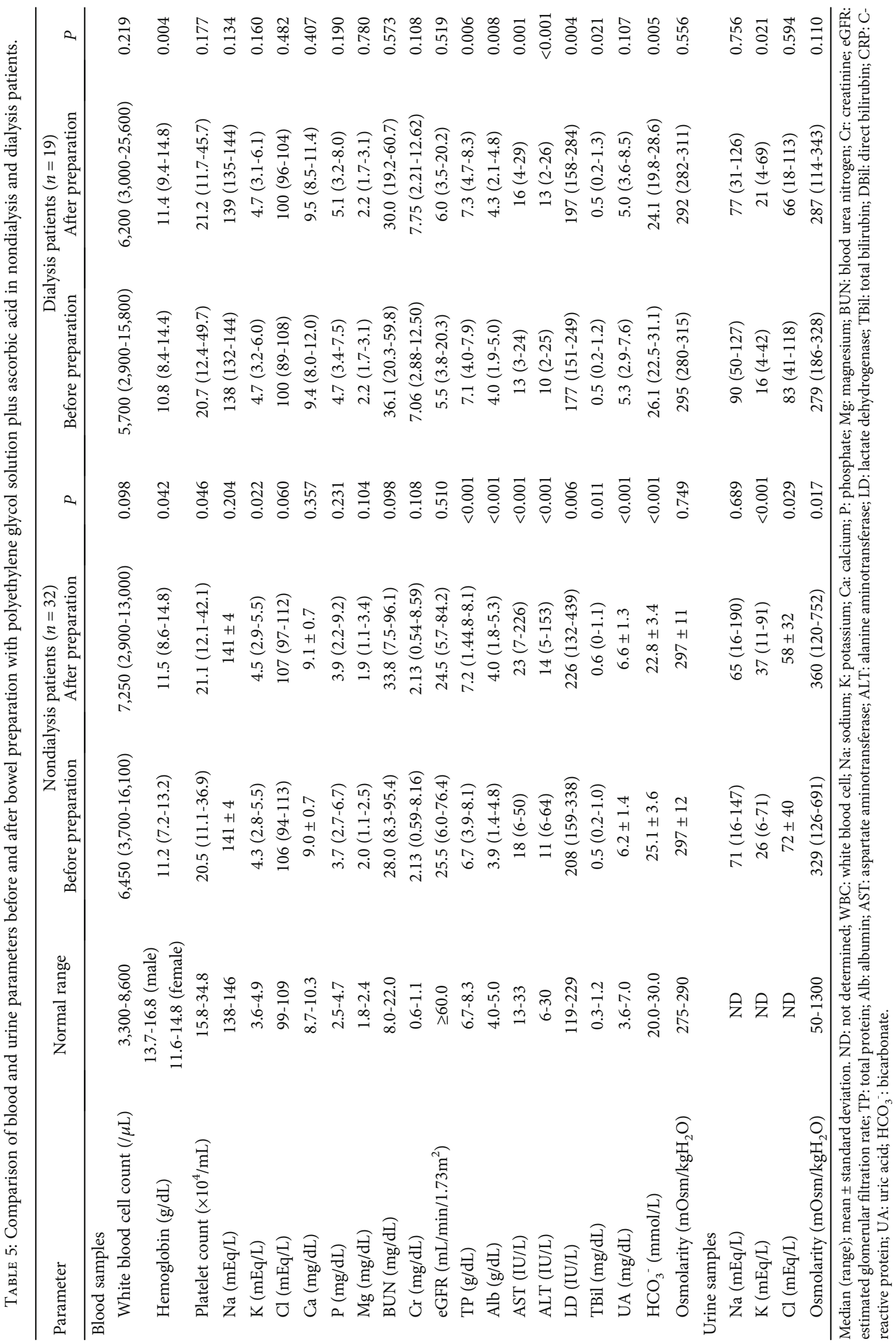

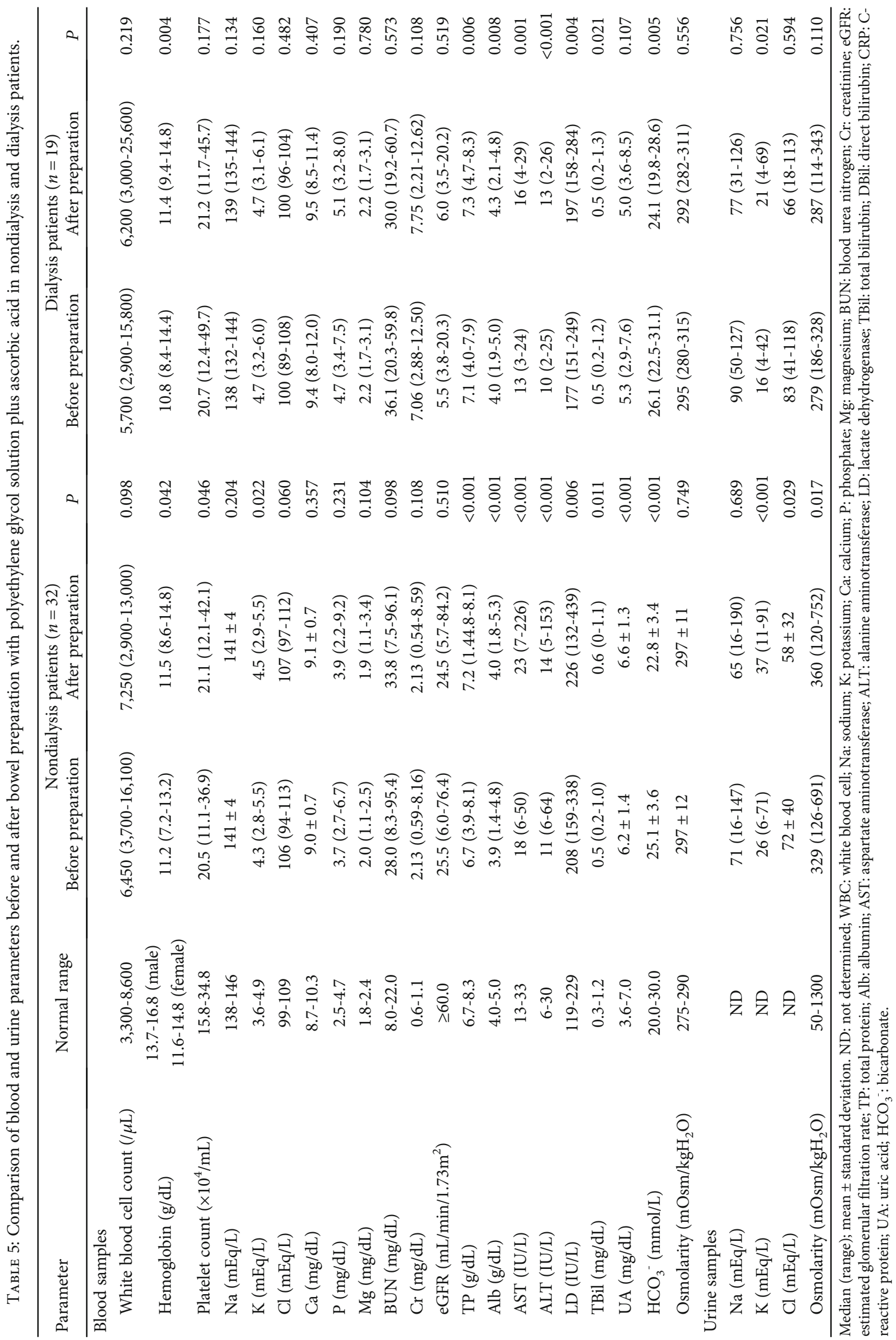


(59.6 mmol) of sodium L-ascorbate per pack as an alkaline agent instead of bicarbonate contained in PEG-ELS because the combination of ascorbic acid and bicarbonate releases carbon dioxide as a byproduct of the acid-base reaction, and $2 \mathrm{~L}$ of PEG-ELS-Asc stimulates $3 \mathrm{~L}$ of defecation accompanied by the loss of bicarbonate. This significant decrease in serum sodium bicarbonate, however, seems clinically irrelevant because of bicarbonate and an electrolyte-water balance parameter; serum osmolarity remained within the normal ranges after colonoscopy in most patients with CKD. A significant increase in AST, ALT, and LD was observed after colonoscopy. Elevated ALT levels were also observed even in patients without comorbidities using both PEG-ELS and PEG-ELS-Asc [11]. These affected hepatic parameters were irrelevant to the use of sedatives and antispasmodics during colonoscopy or endoscopic intervention in the present study. Although these changes remained within the normal ranges in most patients, one patient showed transient abnormal liver function. The mechanism of altered hepatic parameters is still unknown, but it may have been due to transient hypoperfusion. An increase in total bilirubin is considered due to a circadian rhythm or fasting for a long time on an examination day [13]. The important finding of the present study is renal function represented by serum BUN, creatinine, and eGFR, and significant electrolyte disturbance was not observed after bowel preparation, and nondialysis patients did not develop into a dialysis stage. A slight increase in serum potassium and uric acid observed only in nondialysis patients presumably due to dehydration is considered clinically irrelevant. This increase in serum potassium was not associated with the disuse of diuretics or the intake volume of PEG-ELS-Asc. The reason why serum potassium increased only in nondialysis patients is unknown. The urine potassium level was significantly increased in both the nondialysis and dialysis patients, and urine chloride was significantly decreased, and osmolarity was significantly increased only in nondialysis patients, which was considered to compensate for blood changes. The efficacy of the conventional volume of PEG-ELS-Asc in CKD patients was satisfactory because an adequate bowel cleansing score was achieved in $94 \%$ of patients in the present study, which was equivalent to the previous studies in subjects without comorbidities $[3,14]$.

Recently, a 1 L PEG-ELS-Asc solution (Plenvu ${ }^{\circledR}$; Norgine, Harefield, United Kingdom) has been introduced on the market, and it was reported to allow overall cleansing success, high-quality cleansing of the right colon, and tolerability, compared to sodium picosulfate with magnesium citrate [15], 4L PEG preparation, and 2L PEG-ELS-Asc solution [16]. Plenvu ${ }^{\circledR}$ consists of as much as 48.11 grams of sodium L-ascorbate but doses of other elements similar to MoviPrep ${ }^{\circledR}$. Therefore, Plenvu ${ }^{\circledR}$ may also be safe in patients with CKD.

The present study has inherent limitations, including a small sample size of unconsecutive patients, a single-center study without controls, and blood and urine analysis after colonoscopy. Further studies in a larger population are required to confirm our findings. In conclusion, our study suggests the safety and efficacy of bowel preparation with a con- ventional volume of PEG-ELS-Asc for colonoscopy in CKD patients regardless of hemodialysis. Blood and urine examinations before and after colonoscopy may not be routinely monitored when using PEG-ELS-Asc solution (MoviPrep ${ }^{\circledR}$ ).

\section{Abbreviations}

PEG-Asc: Polyethylene glycol plus ascorbate

CKD: $\quad$ Chronic kidney disease.

\section{Data Availability}

The data used to support the findings of this study are included within the article.

\section{Ethical Approval}

This study was registered with the University Hospital Medical Information Network (UMIN000013041).

\section{Disclosure}

EA Pharma Co., Ltd., had no roles in the study design, practice, or analysis of this study, interpretation of the results, or decision to publish.

\section{Conflicts of Interest}

The other authors report conflicts of interest.

\section{Authors' Contributions}

The contributions of the authors involved in this study are as follows: study concept and design, drafting of the manuscript, critical revision of the manuscript for important intellectual content, statistical analysis, and interpretation of data by Naoki Ohmiya and acquisition of data by Naoki Ohmiya, Yoshihito Nakagawa, Noriyuki Horiguchi, Takafumi Omori, Toshiaki Kamano, Kohei Funasaka, Mitsuo Nagasaka, and Tomoyuki Shibata.

\section{Acknowledgments}

The authors thank the staff members of the endoscopy center of Fujita Health University Hospital for data recording. Naoki Ohmiya declares that he received financial support of the operating grant from EA Pharma Co., Ltd.

\section{Supplementary Materials}

Supplementary Table: vital signs before and after 56 cases. (Supplementary Materials)

\section{References}

[1] D. A. Johnson, A. N. Barkun, L. B. Cohen et al., "Optimizing adequacy of bowel cleansing for colonoscopy: recommendations from the US Multi-Society Task Force on Colorectal Cancer," Gastroenterology, vol. 147, no. 4, pp. 903-924, 2014.

[2] F. Radaelli, G. Meucci, G. Imperiali et al., "High-dose senna compared with conventional PEG-ES lavage as bowel 
preparation for elective colonoscopy: a prospective, randomized, investigator-blinded trial," The American Journal of Gastroenterology, vol. 100, no. 12, pp. 2674-2680, 2005.

[3] C. Ell, W. Fischbach, H. J. Bronisch et al., "Randomized trial of low-volume PEG solution versus standard PEG + electrolytes for bowel cleansing before colonoscopy," The American Journal of Gastroenterology, vol. 103, no. 4, pp. 883-893, 2008.

[4] J. X. Wilson, "Regulation of vitamin C transport," Annual Review of Nutrition, vol. 25, no. 1, pp. 105-125, 2005.

[5] I. Fujita, Y. Akagi, J. Hirano et al., "Distinct mechanisms of transport of ascorbic acid and dehydroascorbic acid in intestinal epithelial cells (IEC-6)," Research Communications in Molecular Pathology and Pharmacology, vol. 107, no. 3-4, pp. 219-231, 2000.

[6] N. Yoshida, Y. Naito, T. Murakami et al., "Safety and efficacy of a same-day low-volume $1 \mathrm{~L}$ PEG bowel preparation in colonoscopy for the elderly people and people with renal dysfunction," Digestive Diseases and Sciences, vol. 61, no. 11, pp. 32293235, 2016.

[7] J. M. Lee, B. Keum, I. K. Yoo et al., "Polyethylene glycol plus ascorbic acid for bowel preparation in chronic kidney disease," Medicine (Baltimore), vol. 95, no. 36, article e4755, 2016.

[8] Group KDIGOKCW, "KDIGO 2012 clinical practice guideline for the evaluation and management of chronic kidney disease," Kidney International, vol. 3, pp. 63-72, 2013.

[9] S. Matsuo, E. Imai, M. Horio et al., "Revised equations for estimated GFR from serum creatinine in Japan," American Journal of Kidney Diseases, vol. 53, no. 6, pp. 982-992, 2009.

[10] E. J. Lai, A. H. Calderwood, G. Doros, O. K. Fix, and B. C. Jacobson, "The Boston bowel preparation scale: a valid and reliable instrument for colonoscopy-oriented research," Gastrointestinal Endoscopy, vol. 69, no. 3, pp. 620-625, 2009.

[11] M. Tajika, T. Tanaka, M. Ishihara et al., "A randomized controlled trial evaluating a low-volume PEG solution plus ascorbic acid versus standard PEG solution in bowel preparation for colonoscopy," Gastroenterology Research and Practice, vol. 2015, Article ID 326581, 2015.

[12] N. Ogino, G. Aridome, J. Oshima et al., "Serum albumin concentrations predict hypovolaemia caused by polyethylene glycol plus ascorbic acid prior to colonoscopy in elderly patients," Drugs \& Aging, vol. 33, no. 5, pp. 355-363, 2016.

[13] A. Larsson, M. Hassan, P. Ridefelt, and J. Axelsson, "Circadian variability of bilirubin in healthy men during normal sleep and after an acute shift of sleep," Chronobiology International, vol. 26, no. 8, pp. 1613-1621, 2009.

[14] C. Ell, W. Fischbach, P. Layer, and M. Halphen, "Randomized, controlled trial of $2 \mathrm{~L}$ polyethylene glycol plus ascorbate components versus sodium phosphate for bowel cleansing prior to colonoscopy for cancer screening," Current Medical Research and Opinion, vol. 30, no. 12, pp. 2493-2503, 2014.

[15] S. Schreiber, D. C. Baumgart, J. P. H. Drenth et al., "Colon cleansing efficacy and safety with 1 L NER1006 versus sodium picosulfate with magnesium citrate: a randomized phase 3 trial," Endoscopy, vol. 51, no. 1, pp. 73-84, 2019.

[16] M. Maida, E. Sinagra, G. C. Morreale et al., "Effectiveness of very low-volume preparation for colonoscopy: a prospective, multicenter observational study," World Journal of Gastroenterology, vol. 26, no. 16, pp. 1950-1961, 2020. 